\title{
How COVID-19 is changing rheumatology clinical practice
}

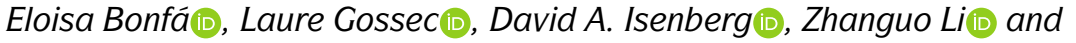 Soumya Raychaudhuri (i)}

Abstract | The emergence of COVID-19 in early 2020 led to unprecedented changes to rheumatology clinical practice worldwide, including the closure of research laboratories, the restructuring of hospitals and the rapid transition to virtual care. As governments sought to slow and contain the spread of the disease, rheumatologists were presented with the difficult task of managing risks, to their patients as well as to themselves, while learning and implementing new systems for remote health care. Consequently, the COVID-19 pandemic led to a transformation in health infrastructures and telemedicine that could become powerful tools for rheumatologists, despite having some limitations. In this Viewpoint, five experts from different regions discuss their experiences of the pandemic, including the most challenging aspects of this unexpected transition, the advantages and limitations of virtual visits, and potential opportunities going forward.

Since the COVID-19 pandemic began, what have been the biggest challenges to managing patients with non-COVID-19 conditions?

Soumya Raychaudhuri. In mid-March 2020, Massachusetts, USA, had about 100 reported cases of coronavirus disease 19 (COVID-19), most emerging from an outbreak from a meeting of pharmaceutical company executives at a Boston hotel in late February ${ }^{1}$. Simultaneously, my colleagues and I were confronted with rapidly emerging data about the asymptomatic spread of this virus ${ }^{2}$. By 13 March, Boston and the surrounding public schools were shutting down, and our research laboratories were directed to work from home except for the most essential functions. At the same time, our outpatient clinic shifted to limit capacity to all but those patients most in need of care. Brigham and Women's Hospital (BWH) made the remarkable switch to making virtual visits available to our patients. Although virtual care worked well for some of our patients, it did mean that we faced unprecedented challenges in taking care of our newest and most active patients. Many of our patients are on immunomodulatory therapies and were appropriately reluctant to travel into health-care facilities, including for diagnostic tests and clinical laboratory monitoring or even infusions. The result was that with the COVID-19 pandemic, many of the tools that we commonly wielded became unwieldy.

This issue was particularly problematic for patients seeing us for a first visit, for patients who were failing to respond to therapies, for patients who needed to be seen urgently for concerning new symptoms or for patients who needed a referral to another specialist for evaluation and work-up of related independent diagnoses. In many instances, we used inadequate temporizing measures rather than a durable solution. For example, some patients with newly diagnosed inflammatory arthritic diseases were prescribed courses of prednisone until an in-person visit became possible. COVID-19 has also taken an emotional toll on our patients, as, like many, they struggled to balance their personal lives as our society shifted towards a lockdown and with the anxiety of a pandemic.

Eloisa Bonfá. On 23 March, 1 week after the first death from COVID-19 was reported in the city of São Paulo, Brazil, a major and difficult decision was taken by the largest tertiary public hospital in Latin America, consisting of 2,400 beds and eight specialized Institutes (Heart and Lung, Orthopaedic, Psychiatry, Children, Cancer, Central, Rehabilitation and Radiology Institutes). As the clinical director of the hospital and one of the coordinators of the COVID-19 crisis Committee, I was involved in the decision to isolate the Central Institute (containing 900 beds) solely for patients with COVID-19 (REF. ${ }^{3}$ ). This decision meant that the other seven Institutes remained at low exposure for COVID-19. All non-COVID patients from our General Tertiary Emergency Unit and from more than 30 specialized ward Units allocated in the Central Institute, including the rheumatology unit, were transferred to these COVID-cold Institutes. Patients from the rheumatology unit were transferred to the Orthopaedic Institute, along with patients from almost all specialized clinical wards. Each specialized ward was allocated to one Unit that had approximately $50 \%$ as many beds as were previously allocated to that ward. Overall, the pandemic resulted in delays in non-emergency hospitalizations.

One main challenge during this period was to divide the team between those who would work in the non-COVID-19 area and those who were recruited to exclusively care for patients with COVID-19 in the isolated COVID-19 Institute. A safe hospitalization flow for inpatients and employee safety was quickly established and upon suspicion of COVID-19, the patient was rapidly transferred to the transition area of the isolated COVID-19 Institute. Another challenge was to increase the number of intensive care unit (ICU) beds available in this Central Institute from 100 to 300 in 2 months. To achieve this goal, we had to convert 34 surgery rooms into 76 ICU beds. During the first 4 months of the pandemic (April-July) in São Paulo, >4,000 patients with severe COVID-19 were hospitalized in the isolated institute, and ICU beds accounted for more than half of these patients. In terms of patients with rheumatic diseases, the number of hospitalizations decreased by $\sim 40 \%$ compared with the same period in the previous year and the number of patients in our Rheumatology outpatient clinics decreased by $\sim 34 \%$, 
reducing from a mean of $\sim 1,730$ patients per month to $\sim 1,148$ patients per month. The Rheumatology Biological Center, a separate Unit dedicated exclusively to patients under biologic therapy, remained opened during the pandemic, and the number of appointments reduced by only $\sim 16 \%$ compared with the same period in the previous year.

\section{Zhanguo Li. As a rheumatologist} practicing at Peking University People's Hospital, Beijing, the biggest challenge during the COVID-19 pandemic has been how to manage patients with rheumatic diseases remotely using online as WeChat) or telephone calls, because the patients simply could not physically attend the hospital. This alternative access to care was unprecedented and was previously even prohibited by our medical systems and insurance policies. The situation was extremely challenging for rheumatologists and patients for quite a few months, as rheumatologists had no existing online, regulated system for prescribing treatments. Consequently, the ceasing of medication or inappropriate self-management occurred in many patients across the country, resulting in flares of disease in some patients. systems, social media platforms (such

David Isenberg. Managing patients with serious autoimmune rheumatic diseases (who are often on steroids, immunosuppressives and/or biologics) who you cannot see and examine and do blood tests on has been a huge challenge. It is clear that many patients who have been carefully shielding have not wanted to come to hospital (at the University College Hospital, situated in the centre of London) and some have clearly tried hard to deny (to themselves as well as to their physicians) the fact that their underlying disease was getting worse. We had a particularly troubling time 2 months into the pandemic when, in a period of about 1 week in April, we had to admit six patients with systemic lupus erythematosus (SLE) who were experiencing acute flares - three of whom went straight into the ICU and two of whom died.

Laure Gossec. An overall and overarching challenge to my practice as an academic full-time rheumatologist at Sorbonne Université and Pitié-Salpêtrière Hospital, Paris, France, was my inner turmoil. When I was young, I spent a few months doing volunteer medical work in a developing country, but for me, this role led to less personal risk than the current pandemic, especially as personal protection equipment

\section{The contributors}

Eloisa Bonfá is a full professor of rheumatology and the clinical director of the largest tertiary public hospital of Latin America. Her main clinical and research interests are systemic lupus erythematosus and autoimmunity, with relevant contributions in the fields of autoantibodies, vaccines and drug monitoring in autoimmune diseases. She graduated at the University of São Paulo Medical School, Brazil, and undertook specialist training in rheumatology in the same university followed by a 4-year rheumatology research fellowship at the Hospital for Special Surgery, New York.

Laure Gossec is a professor of rheumatology at Sorbonne Université and Pitié-Salpêtrière Hospital, Paris, France. She has a half-time clinical position where she mainly sees patients with inflammatory arthritis, and a half-time teaching and research position. Her main research interests are patientreported outcomes and quality of life, as well as e-health and big data in psoriatic arthritis, spondyloarthritis and rheumatoid arthritis and she has authored more than 350 papers. She is a past-chair of the epidemiology standing committee of EULAR.

David Isenberg is the Academic Director of Rheumatology at University College London, UK. He has run both general and autoimmune rheumatic disease clinics for over 30 years. His major research interests are in the structure, function and origin of autoantibodies and improving the assessment of patients with autoimmune rheumatic diseases.

Zhanguo $\mathrm{Li}$ is a professor and head of the department of rheumatology and immunology at the Peking University People's Hospital, China. He is the past president of APLAR, and the president of the Clinical Immunology Committee at the Chinese Society for Immunology. He is Editor-in-Chief of the Chinese Journal of Rheumatology. His research interests are the mechanisms and immune therapy of rheumatic diseases, including rheumatoid arthritis and systemic lupus erythematosus.

Soumya Raychaudhuri is a Professor at Harvard Medical School, and a practicing rheumatologist at the Brigham and Women's Hospital Arthritis Center. He is also appointed at the Broad Institute, and the University of Manchester. He spends most of his time running a lab that is focused on defining mechanisms of disease in rheumatoid arthritis, and other immune-mediated diseases, using computational biology, genetics and functional genomics. was scarce or lacking, and I feared bringing COVID-19 back home. This situation challenged my conviction that my job as a rheumatologist is the best in the world!

For me, the second biggest challenge to managing my (non-COVID) patients over the past months has been my fear of putting them at risk through my prescriptions. I mainly see patients with inflammatory arthritis, most of whom are treated with biologics or other targeted therapies. Initially, we had no information as to the potential risk associated with such treatments, in terms of increasing the risk of or severity of COVID-19. Thus, whereas I have always prescribed such treatments with the conviction of helping my patients, the challenge here is a profound rethinking of the benefit-to-risk balance of my prescriptions.

How have your clinical and research activities changed? What adaptations have you put in place?

Eloisa Bonfá. For the first time, the Rheumatology Outpatient Clinics of our Hospital provided virtual care over the phone to define which patients could have their visit postponed, which patients needed a change in prescription or which patients had to come to the clinic for an appointment. Postponing all previously scheduled rheumatology outpatient appointments was a challenging task owing to the large number of patient appointments per week (approximately 400), and it required a team of staff fully dedicated to this assignment. Those health-care workers who were at a high risk of severe illness from COVID-19 were selected for this job. This procedure required several adaptations for the medical staff and patients due to the lack of previous experience with virtual care, as telehealth was only endorsed by the Federal Council of Medicine during the pandemic ${ }^{4}$. Several measures of care and risk assessment were established for patients who were required to come into the clinic for an appointment, such as screening for COVID-19 symptoms at entry and at the reception as part of the routine clinical assessment. Patients were recommended not to attend a face-to-face appointment if they had any symptoms of COVID-19. Other adaptations included reviewing appointment scheduling, physical distancing in waiting rooms, hand hygiene care and appropriate personal protective equipment. Mask wearing is still mandatory in Brazil for any outside activity during the pandemic ${ }^{5}$ and is also compulsory for patients during appointments. 
Zhanguo Li. To adapt to the totally unexpected changes to clinical practice, one option in my department of the People's Hospital was to set up a consultant team consisting of 26 rheumatologists to provide medical service free to patients with rheumatic diseases, supported technically by an internet company. It was the first rheumatologist team to provide such support to patients in the country. Many patients nationwide were helped by this group over a 2-month period, from early February to late March 2020.

In addition, we used a previously developed smartphone application (smart system of disease management (SSDM)) as a patient self-care instrument to evaluate disease activity and remind patients to contact rheumatologists. The SSDM system was designed for a research project ${ }^{6}$, and the clinical value was also clearly shown in the patients who used this SSDM system during the initial months of the COVID-19 pandemic.

David Isenberg. My practice has changed completely. During the first 3 months of the pandemic, no routine appointments were offered (although an emergency clinic once a week was available) so that all outpatient consultations took place over the phone or occasionally by video conferencing. For patients with longstanding, well-established disease and on low or moderate doses of steroids and immunosuppressives, I was reasonably content to miss seeing the patients at routine follow-up appointments, but increasingly I have become concerned about the inadequacies of what can be done when not seeing patients face-to-face.

Among the pleasures and responsibilities of running clinics in an academically inclined institution are doing research and educating both undergraduate and postgraduate students. The introduction of more remote patient assessment has had, and will always have, a detrimental effect on both. It will be harder to recruit patients to trials. We cannot, for example, perform ACR20, ACR50 or ACR70 assessments of our patients with rheumatoid arthritis or British Isles Lupus Activity Group (BILAG) assessments of our patients with SLE, to help determine their eligibility for a clinical trial. The patient cannot agree to have their blood taken remotely for a project. Likewise, teaching opportunities are restricted if we cannot, for example, demonstrate the use of the cross fluctuation test to show fluid in the knee of a patient, identify an enlarged liver or spleen or identify an extensor plantar response. These problems will obviously be detrimental for patient care too.
Laure Gossec. My professional life has profoundly changed since February. My research activities usually involve very frequent travels to other countries, which have completely stopped since February. My academic work as a professor of rheumatology involves face-to-face interactions with students, which likewise have disappeared completely and have been replaced (partly) by online courses, which are by essence much less interactive.

As regards my clinical work, my practice has changed because the hospital has become a place of dread and doom. My patients with inflammatory arthritis do not want to come to the hospital anymore, and I myself feel reluctant to ask them to come. For this reason, for 3 months, all of my patient clinics were switched to teleconsultation, where no physical examination is possible and where the quality of care is lower. In the hospital, instead of accommodating patients with severe rheumatic diseases, our beds were taken over for patients with non-rheumatic diseases, for whom my added value and competency is much lower.

One of the fun and interesting parts of my work is interactions within the medical and non-medical team as well as with colleagues outside of rheumatology (such as through staff meetings). Most of this social interaction has now disappeared, replaced somewhat by e-mail exchanges.

Soumya Raychaudhuri. I spend most of my time running a research lab in an academic setting. That part of my life has completely changed. Like many workplaces, we have moved almost entirely to virtual work environments. Hence, research and education has become much less interactive and we have had to shift our culture to accommodate this major change.

My clinical practice is within the BWH Arthritis Center, which is a large clinic that hosts 30,000 patient visits per year. My practice specifically has shifted to include more virtual visits and fewer in-person visits. From March to July, my practice was almost entirely virtual.

For in-person visits, to reduce the risk of infection for our staff and our patients, the BWH Arthritis Center has made dramatic changes in the way we interact with each other and with our patients, the flow of patients in and out of the clinic and the clinic rooms, how clinic rooms are turned over and many other components. The changes have been well executed and have affected every aspect of our clinical experience. The result is that I feel confident to encourage my patients to come in and see me, especially when so many of them are worried about COVID-19 and the personal risk to themselves. Although these changes are essential to our ability to see patients in person, they do make the experience of being a doctor somewhat less personal. Implementing social distancing has meant that I see fewer colleagues and staff. It also means that many of the spouses and family members that often accompanied my patients are no longer present. I no longer greet my patients in a crowded waiting room, rather they are brought in from an empty waiting room. Masks are essential to protect our patients, especially those on immunomodulatory therapies, but they do make non-verbal cues harder to glean. Overall, in-person visits continue to be essential, but they do not feel quite as warm or friendly.

On the other hand, virtual visits have been much more effective than I might have anticipated. Our clinical infrastructure has enabled video visits, which have proven to be far more productive than a simple phone call. The video visits are very practical and effective for my longstanding patients who are doing well on established therapeutics. Previously, some patients who live further away might have taken a day off to drive into Boston - in some instances from out of state - for a physical visit. For some of these patients, the ability to do a visit virtually has saved them valuable time. The virtual visit is often more efficient as visits can be easily started and ended, and the next visit can be started immediately. But the virtual visit has definite limitations. Most obviously, the inability to do an in-person physical examination and joint examinations cannot be reproduced via video. The exam is essential for assessing our patient's disease activities or making diagnoses, and taking care of new patients or patients with active disease can hence be really challenging. Video visits expose the digital divide of our society, and some of our patients are unable to fully take advantage of our infrastructure, especially those who are of fewer means, have poorer internet access or are older and less comfortable with technology.

\section{[0]} Will COVID-19 change your clinical and research activities for good, or will you return to business as usual once the situation is back to 'normal'?

David Isenberg. Although apocryphal, there is a story that the then Chinese Premier Zhou Enlai, when asked by Henry Kissinger, Richard Nixon's secretary of state, 
for his opinion on the effects of the French Revolution, replied "too early to say". I think the same is true for assessing the long-term effects of COVID-19. The pandemic has highlighted the value (at least in the short term) of fully electronic record systems, which makes it possible to see patient records, including letters, imaging and blood test results, remotely. I can certainly envisage that some routine follow-up appointments can be undertaken remotely and safely (provided local blood tests can be done), which may well reduce the numbers of patients attending specialist clinics.

Eloisa Bonfá. Engaging back to 'normal' activities will take time and it will probably have to wait for a vaccine. Until then, all adaptations and risk assessments will remain. But one of the major gains the COVID-19 pandemic will bring is the consolidation of telemedicine and televisits in the care of patients. Taking into account that many patients with rheumatic diseases have mobility difficulties, telehealth will provide an alternative approach to the care of these patients, when possible. Furthermore, in a large city such as São Paulo, with chaotic traffic and long distances, the possibility of avoiding public transportation, not only to prevent the spread of COVID-19 but also to avoid other issues beyond the pandemic, will be more convenient for the patient.

Zhanguo Li. COVID-19 has certainly changed rheumatology practice. Although the patient volume has now returned to normal in China, the demographics of patients attending outpatient clinics have altered in terms of disease severity and distance of travel. Patients with mild diseases who live in remote areas now tend to see their local doctors, rather than come to rheumatology centres.

Laure Gossec. At this stage, I do not really foresee the situation ever fully getting back to normal. It seems to me that social distances will be increased for a long time. In France, we usually hug and kiss a lot, which I do not think will go back to normal anytime soon. As for my professional life, I do not foresee going back to my previous rate of travel related to my research activities. I also think that medical teaching will be profoundly modified now with much more online resource use and much less face-to-face teaching. From that point of view, we were quite late in France in adopting these teaching methods, and this pandemic might well be an opportune moment for this change. As for my patients,
I do think the situation will mostly go back to normal, as my clinics require the use of physical examinations and ultrasonography. I am planning to keep around $10 \%$ of consultations online for patients in the long term.

Soumya Raychaudhuri. I think that some of the changes will be here to stay. Boston is a challenging city for many of our patients to get in and out of, particularly those who are coming from far away, or for those for whom driving or navigating public transportation is hard. For these patients, especially for routine follow-up visits, a virtual visit can offer real advantages. There are patients all over New England who would benefit from access to a referral centre. I can imagine if our institution or others are able to build a great virtual care infrastructure, we could be in a position to expand the scope of patients who our physicians are connecting with and caring for.

\section{(1)}

If temporary adaptations are to become permanent, what barriers need to be overcome?

\section{Laure Gossec. Barriers to online} consultations include poor access to the internet for some patients, low-quality internet connection on either side, a lack of user-friendly medical files and also a psychological reluctance from patients regarding online consultations (most patients prefer to see me face-to-face).

The wearing of masks is also a barrier to my clinical practice. It hinders the interactions with my patients, which makes shared decision-making (probably the most rewarding part of my clinics) more difficult. Will it be that masks will push us back in time, to paternalistic prescriptions? Who can say?

Zhanguo Li. Current barriers are the lack of a 'telehealth' and medical support system for patient care, which can facilitate patients and doctors in terms of consultations, efficient follow-up and clinical studies. If a second wave of COVID-19 comes, we will face the same difficulty as we had a few months ago.

Eloisa Bonfá. The most important adaptation is consolidation of the regulatory framework for telemedicine in Brazil, including reimbursement for this activity. Another notable barrier that is expected is the serious economic crisis resulting from the COVID-19 pandemic that will limit investment resources in all areas including health. This limitation of resources will hinder the development and implementation of innovations. Hopefully, increased solidarity, a hallmark of this crisis, and regional cooperation will help to overcome the challenges we will have during reconstruction.

Soumya Raychaudhuri. I think telemedicine and virtual medical care could become really powerful tools for the right patient with the right infrastructure. I think that we need to make sure that our patients have access to a proper IT infrastructure to mitigate access issues. If language is a barrier, we need to have a means of enabling translation services during our virtual visits. To realize the full potential of virtual care, we need to be able to arrange services and testing for our patients within their communities. After the visit, having an integrated health-care system that enables seamless data transfer is essential. With such an integrated health-care system, arranging imaging, lab work, therapeutic infusions and other services near to home becomes possible without cumbersome administrative barriers. Currently, for my more distant patients, I often need to bring them into Boston for tests and services. In many cases, they have alternative facilities near to their home, but those facilities are not connected to our system, and arranging local testing and services is challenging without extensive administrative effort.

David Isenberg. I anticipate that it will be even more important to stress to patients, if their disease is worsening and they have not been seen by a physician (or nurse), that they must contact the hospital and arrange a face-to-face appointment as soon as possible. From the administrative point of view, there will need to be greater flexibility about determining whether patients are to be seen face-to-face or via a telephone consultation. Closer links with general practices will also be necessary as, in my experience, some general practitioners have been reluctant to take on routine monitoring of patients on immunosuppressive medication.

What other opportunities lie ahead for transforming rheumatology practice?

Zhanguo Li. Many opportunities lie ahead, as long as we focus on the needs of patients and rheumatologists. Undoubtedly, more patient-associated and doctor-associated activities will be held online, providing opportunities for patient education and 
virtual conferences, although patients with severe or difficult-to-treat disease will still need face-to-face appointments with their rheumatologist.

Laure Gossec. Improving access to best care, through online consultations but also by improving the patient trail (that is, the way in which patients first see their general practitioner before being referred to a rheumatologist) and decreasing the delay before a consultation, is a priority. Better use of online resources and maybe of rheumatology nurses, if they are allowed to play a bigger role in France, are options to move forward, which may be facilitated by the COVID-19 pandemic.

Soumya Raychaudhuri. The implementation of effective virtual visits will be really powerful for rheumatology. The need for an in-person visit will always be there, especially for patients with very active disease or for new patients with uncertain diagnoses. But for patients who we know well, managing them to some extent virtually will have great value. I think in practice these are the patients we talk to on the phone informally and e-mail with. So, having a formal mechanism to take care of them will be beneficial to them and to us.

David Isenberg. By doing more telephone consultations and reducing the numbers of patients attending clinics face-to-face, it should be possible to reduce the waiting times for patients referred to rheumatologists. I am, though, becoming increasingly concerned about the 'downsides' of what has happened in the past 6 months, notably the missed occurrences of increased disease activity in patients, the loss of educational opportunities for physicians and the difficulties in undertaking translational research.

Eloisa Bonfá. Innovations associated with self-care, including smartphone apps and wearable technologies, consolidated during the pandemic, are interesting alternatives for the management of several chronic conditions and will certainly also be useful for patients with rheumatic diseases. Above all, there is no way back and the acceleration of digital transformation and the improvements in internet speed that occurred during the pandemic will continue and will transform our lives. This change will provide new opportunities for physicians to update their knowledge on the field and for continuing medical education online, without the need for physical travel. In addition, for organizations, a new way of dealing with administrative work took place with changes in workflows, including replacement of meetings with e-mails, increased working from home and accelerated automation that will forever change the way we work.

Eloisa Bonfá iD ${ }^{1 凶}$, Laure Gossec (iD) ${ }^{2,3 凶}$, David A. Isenberg (iD ${ }^{4 凶}$, Zhanguo Li (iD ${ }^{5 凶}$ and Soumya Raychaudhuri (iD ${ }^{6,7,8}$ 区

${ }^{\prime}$ Rheumatology Division, Hospital das Clinicas HCFMUSP, Faculdade de Medicina, Universidade de São Paulo, São Paulo, SP, Brazil.

${ }^{2}$ Sorbonne Université, Institut Pierre Louis d'Epidémiologie et de Santé Publique, Paris, France. ${ }^{3}$ Department of rheumatology, AP-HP, Pitié Salpêtrière Hospital, Paris, France.

${ }^{4}$ Centre for Rheumatology/Division of Medicine, University College London, London, UK.

${ }^{5}$ Peking University People's Hospital, Beijing, China.

${ }^{6}$ Brigham and Women's Hospital and Harvard Medical School, Boston, MA, USA
${ }^{7}$ Broad Institute of MIT and Harvard, Cambridge, MA, USA.

${ }^{8}$ Manchester Academic Health Science Centre, University of Manchester, Manchester, UK.

凶e-mail: eloisa.bonfa@hc.fm.usp.br; laure.gossec@ aphp.fr; d.isenberg@ucl.ac.uk; li99@bjmu.edu.cn; soumya@broadinstitute.org

https://doi.org/10.1038/s41584-020-00527-5

Published online 2 November 2020

1. Stockman, F. \& Barker, K. How a Premier U.S. Drug Company Became a Virus 'Super Spreader'. The New York Times https://www.nytimes.com/2020/04/12/us/ coronavirus-biogen-boston-superspreader.html (2020).

2. Rothe, C. et al. Transmission of 2019-nCoV infection from an asymptomatic contact in Germany. N. Engl. J. Med. 382, 970-971 (2020).

3. Miethke-Morais, A. et al. Overcoming barriers to providing comprehensive inpatient care during the COVID-19 pandemic. Clinics 75, 2100 (2020).

4. de Britto Ribeiro, M. L. Letter No.1756/2020, Federal Council of Medicine, Brasilia (Mauro Luiz de Britto Ribeiro to Luiz Henrique Mandetta). https://portal.cfm. org.br/images/PDF/2020_oficio_telemedicina.pdf (2020).

5. Doria, J. Decree No. 64.959, Legislative Assembly of the State of São Paulo, São Paulo https://www.al.sp. gov. $\mathrm{br} /$ norma/?id=193701 (2020).

6. Zhong, J. et al. COVID-19 in patients with rheumatic disease in Hubei province, China: a multicentre retrospective observational study. Lancet Rheumatol. 2, e557-e564 (2020).

\section{Acknowledgements}

E.B. has received grants from the Conselho Nacional de Desenvolvimento Científico e Tecnológico (\#305068/2014-8) and Fundação de Amparo à Pesquisa do Estado de São Paulo (FAPESP) (\#2015/03756-4). Z.L. would like to thank all the rheumatologists in his group, at the Peking University People's Hospital, for their contributions to patient care, and would also like to thank Dr. Fei Xiao and his colleagues for providing SSDM system support during the COVID-19 pandemic.

\section{Competing interests}

L.G. receives research grants from Amgen, Galapagos, Janssen, Lilly, Pfizer, Sandoz and Sanofi, and receives consulting fees from AbbVie, Amgen, BMS, Celgene, Gilead, Janssen, Lilly, Novartis, Pfizer, Samsung Bioepis, Sanofi-Aventis and UCB, all unrelated to the present paper. S.R. is an employee of Brigham and Women's Hospital, and has recently served as a consultant for AbbVie, Biogen, Gilead, Merck and Pfizer. He is a founder of Mestag. He currently receives research funding from Biogen. E.B., D.A.I. and Z.L. declare no competing interests.

Publisher's note

Springer Nature remains neutral with regard to jurisdictional claims in published maps and institutional affiliations.

(c) Springer Nature Limited 2020 